\title{
Epiligrin, the Major Human Keratinocyte Integrin Ligand, Is a Target in Both an Acquired Autoimmune and an Inherited Subepidermal Blistering Skin Disease
}

\author{
Nouha Domloge-Hultsch, “ W. Ray Gammon, ${ }^{*}$ R. A. Briggaman, ${ }^{*}$ Susana G. Gil, ${ }^{\star}$ William G. Carter, ${ }^{\star}$ and Kim B. Yancey \\ ${ }^{*}$ Department of Dermatology, Uniformed Services University of the Health Sciences, Bethesda, Maryland 20814; ${ }^{\ddagger}$ Department \\ of Dermatology, University of North Carolina, Chapel Hill, North Carolina 27514; and ${ }^{\S}$ Fred Hutchinson Cancer \\ Research Center and Department of Pathobiology, University of Washington, Seattle, Washington 98104
}

\begin{abstract}
Epiligrin, the major component of human keratinocyte extracellular matrix, serves as the preferred integrin ligand for $\alpha_{3} \beta_{1}$ in plasma membranes and focal adhesions, and colocalizes with $\alpha_{6} \beta_{4}$ in hemidesmosomes. In human skin, epiligrin is found in the lamina lucida subregion of epidermal basement membrane, where it is thought to be associated with anchoring filaments. We have identified three patients with an acquired mucosal predominant subepidermal blistering disease who have IgG anti-basement membrane autoantibodies that bind the lamina lucida/lamina densa interface of epidermal basement membrane, stain cultured human keratinocyte extracellular matrix, and immunoprecipitate disulfide linked polypeptides of $\mathbf{1 7 0}$, 145,125 , and $95 \mathrm{kD}$ in human keratinocyte culture media in a pattern identical to that of P1E1, a murine monoclonal antiepiligrin antibody. Comparative immunoprecipitation studies of patient sera, P1E1, and GB3 monoclonal antibody show that epiligrin is identical to the antigen (i.e., BM600 or GB3 antigen) previously reported to be absent from the skin of patients with lethal junctional epidermolysis bullosa, an inherited subepidermal blistering disease. Moreover, skin from a fetus with this disease shows no evidence of reactivity to patient antiepiligrin autoantibodies or P1E1. These studies show that antiepiligrin autoantibodies are a specific marker for a novel autoimmune blistering disease and that the epidermal basement membrane antigen absent in patients with lethal junctional epidermolysis bullosa is epiligrin. (J. Clin. Invest. 1992. 90:1628-1633.) Key words: epiligrin • integrin • autoimmunity • bullous skin diseases $\bullet$ epidermolysis bullosa
\end{abstract}

\section{Introduction}

The stratified squamous epithelium of human epidermis is derived from a proliferating basal keratinocyte cell layer that is attached to the underlying epidermal basement membrane (BM). ${ }^{1}$ Adhesion of basal keratinocytes to epidermal BM is

1. Abbreviations used in this paper: $\mathrm{BM}$, basement membrane; ECM, extracellular matrix; LJEB, lethal junctional epidermolysis bullosa.

Address all correspondence to Kim B. Yancey, M.D., Department of Dermatology, B4080 USUHS, 4301 Jones Bridge Road, Bethesda, MD 20814-4799. 1992.

Received for publication 16 April 1992 and in revised form 26 June

The Journal of Clinical Investigation, Inc.

Volume 90, October 1992, 1628-1633 critical for maintenance of epithelial cell polarization, differentiation, morphogenesis, and wound healing. Basal keratinocyte adhesion to epidermal $\mathbf{B M}$ is mediated by integrin receptors $\alpha_{3} \beta_{1}$ in plasma membranes and focal adhesions, as well as $\alpha_{6} \beta_{4}$ receptors in hemidesmosomes $(1,2)$. While these integrin receptors have traditionally been thought to bind laminins in epidermal BM, the latter are poor ligands for adhesion of cultured human keratinocytes in vitro. Recently, Carter et al. have characterized a human keratinocyte extracellular matrix (ECM) glycoprotein complex termed epiligrin that is the major component of keratinocyte ECM and that serves as the preferred ligand for $\alpha_{3} \beta_{1}$ in plasma membranes and focal adhesions, and colocalizes with $\alpha_{6} \beta_{4}$ in hemidesmosomes (2). In human skin, epiligrin is found in the lamina lucida subregion of epidermal BM adjacent to $\alpha_{3} \beta_{1}$ and $\alpha_{6} \beta_{4}$ in basal keratinocytes. As the major keratinocyte integrin ligand, epiligrin is thought to play a key role in the attachment of the epidermis to BM.

We have identified three patients with an acquired autoimmune blistering disease characterized by separation of the epidermis from BM who have IgG anti-BM autoantibodies that bind human skin, cultured human keratinocyte ECM, and epiligrin in human keratinocyte culture media in a pattern identical to that of P1E1, a murine monoclonal antiepiligrin antibody. Furthermore, comparative immunoprecipitation studies show that keratinocyte epiligrin is identical to BM600, an epidermal BM antigen known to be absent from the skin of infants with a lethal inherited blistering disease (lethal junctional epidermolysis bullosa [LJEB]), in which the epidermis does not adhere to $\operatorname{BM}(3,4)$. These studies demonstrate that epiligrin plays a key role in an acquired autoimmune and a lethal inherited blistering skin disease that are both characterized by defective attachment of epidermis to BM.

\section{Methods}

Patients. Three patients (ages 29,54 , and $72 \mathrm{yr}$ ) with a mucosal predominant, subepidermal blistering disease with clinical features resembling cicatricial pemphigoid $(5,6)$ are included in this study. All patients demonstrated involvement of oromucosal, nasopharyngeal, genital, and/or ocular mucous membranes, as well as occasional skin lesions. Direct immunofluorescence microscopy of normal perilesional skin or mucous membrane samples from these patients shows continuous deposits of IgG and C3 in epidermal BM. Samples of normal fetal skin and skin from a fetus with LJEB were provided by associates (see Acknowledgments) and used in comparative immunostaining experiments, as described below.

Reagents. FITC-conjugated goat anti-human IgG, IgA, IgM, IgE, or fibrinogen (Tago, Inc., Burlingame, CA), peroxidase-conjugated 
goat anti-human IgG (Tago, Inc.), peroxidase- and FITC-conjugated goat $\mathrm{F}\left(\mathrm{ab}^{\prime}\right)_{2}$ anti-mouse IgG ( Tago, Inc.), murine monoclonal antihuman $\operatorname{IgG}_{1}$ (Southern Biotechnology Associates, Birmingham, AL), GB3 murine monoclonal antibody (Accurate Chemical \& Science Corp., Westbury, NY), P1E1 murine monoclonal antiepiligrin antibody (2), murine monoclonal antitenascin antibody (2), and protein A-bearing, formalin-fixed staphylococci (Pansorbin; Calbiochem Behring Corporation, San Diego, CA) were used, as described below.

Cell cultures. Human neonatal foreskin keratinocytes (Clonetics Corp., San Diego, CA) and the A-431 human epidermoid carcinoma cell line (American Type Culture Collection, Rockville, MD) were grown in appropriate media at $37^{\circ} \mathrm{C}$ in humidified $5 \% \mathrm{CO}_{2}$, as previously described (7).

Immunofluorescence studies. 3-mm punch biopsy samples of normal human skin were incubated in $1 \mathrm{M} \mathrm{NaCl}$ containing $1 \mathrm{mM}$ EDTA $1 \mathrm{mM}$ PMSF, and $25 \mathrm{mM}$ Tris- $\mathrm{HCl}(\mathrm{pH} 7.4)$ for $72 \mathrm{~h}$ at $4^{\circ} \mathrm{C}$ to produce a substrate containing a cleavage plane between bullous pemphigoid antigen (epidermal side) and laminin (dermal side) (8). Such $1 \mathrm{M}$ $\mathrm{NaCl}$ split skin serves as a sensitive substrate for detection of antibodies directed against subregions of epidermal BM. Cryostat sections $(6 \mu \mathrm{m})$ of $1 \mathrm{M} \mathrm{NaCl}$ split skin were incubated with serial dilutions of $(a)$ patient serum; $(b)$ normal human serum (negative control); $(c)$ a reference serum sample from a patient with bullous pemphigoid (positive control); (d) P1E1 murine monoclonal antiepiligrin antibody; or $(e)$ murine monoclonal anti-human $\mathrm{IgG}_{1}$ antibody (negative control). Skin sections were next washed three times with PBS, stained with FITC-conjugated goat anti-human (or where appropriate, antimouse) IgG, washed three times again with PBS, and then examined by immunofluorescence microscopy (9). Human keratinocytes and A-431 cells were grown on glass slides (Lab-Tek Division, Miles Laboratories, Inc., Naperville, IL) for $48 \mathrm{~h}$ in their respective standard media, fixed with $2 \%$ formaldehyde in PBS, and then incubated with serum from a representative patient, normal human serum (negative control), P1E1, or murine monoclonal anti-human $\mathrm{IgG}_{1}$ antibody (negative control) for $30 \mathrm{~min}$ at room temperature. Cells were washed three times with PBS then stained, washed again, and examined in the same manner as indirect immunofluorescence microscopy studies of 1 $\mathrm{M} \mathrm{NaCl}$ split skin (described above).

Immunoperoxidase studies. Cryostat sections $(6 \mu \mathrm{m})$ of skin from a human fetus with LJEB and skin from a normal human fetus were reacted with serum from a representative patient, normal human serum (negative control), P1E1 murine monoclonal antiepiligrin antibody, or monoclonal antitenascin antibody (positive control), washed, and then developed with the appropriate peroxidase-conjugated second-step antibodies, as previously described (2).

Immunoelectron microscopy. Direct and indirect IgG immunoelectron microscopy was performed as previously described $(10,11)$. In brief, a multistep immunoenzyme technique was used to characterize in situ immunoreactants in patient skin samples, as well as IgG antiepidermal BM reactivity of patients' sera, normal human serum (negative control), P1E1, and murine monoclonal anti-human $\operatorname{IgG}_{1}$ antibody (negative control).

Biosynthetic radiolabeling of human keratinocyte culture media. Subconfluent monolayers of human keratinocytes (to avoid confluence-associated differentiation changes that occur in epithelial cell cultures) in methionine-free media (KGM;Clonetics Corp.) were metabolically labeled with $\left[{ }^{35} \mathrm{~S}\right]$ methionine $(50 \mu \mathrm{Ci} / \mathrm{ml}$; specific activity $\sim 1100 \mathrm{Ci} / \mathrm{mmol}$, New England Nuclear, Boston, MA) for $24 \mathrm{~h} \mathrm{(7,}$ 12). Recovered culture media was made $1 \mathrm{mM}$ in EDTA and PMSF, centrifuged at $750 \mathrm{~g}$ for $10 \mathrm{~min}$, recovered, dialyzed extensively against $0.01 \mathrm{M}$ Tris- $\mathrm{HCl}, 0.15 \mathrm{M} \mathrm{NaCl}, \mathrm{pH} 7.4$ (Tris-buffered saline [TBS]) at $4^{\circ} \mathrm{C}$, and then stored at $-70^{\circ} \mathrm{C}$.

Immunoprecipitation. The protein content of radiolabeled human keratinocyte culture media aliquots was estimated by trichloroacetic acid precipitation and used to determine equal sample inputs for immunoprecipitation experiments. Immunoprecipitation experiments were performed as previously described $(7,12,13)$. In brief, aliquots of radiolabeled media were preabsorbed with normal human serum (or normal mouse and rabbit serum in two-step immunoprecipitation studies using murine monoclonal antibodies and rabbit anti-mouse IgG), twice mixed with freshly washed Pansorbin, then incubated overnight at $4^{\circ} \mathrm{C}$ with $10 \mu \mathrm{l}$ of serum (patient or control subjects) or murine monoclonal antibodies. Freshly washed Pansorbin (200 $\mu \mathrm{l}$ of a $10 \%$ wt $/$ vol suspension ) was added to each sample for $1 \mathrm{~h}$ at $4^{\circ} \mathrm{C}$. Pansorbin aliquots were then washed three times with TBS containing $0.3 \%$ Triton $\mathrm{X}-100,0.3 \%$ sodium deoxycholate, $0.3 \mathrm{mM} \mathrm{NaCl}, 0.1 \% \mathrm{BSA}$, three times with the same buffer without BSA, and once with half ionic strength buffer without BSA. Pansorbin, immunoglobulin, and antigens were dissociated in $100 \mu \mathrm{l}$ of $2 \% \mathrm{SDS} \pm 0.1 \mathrm{M}$ DTT at $100^{\circ} \mathrm{C}$ for 2 min. Immunoprecipitation samples were recovered and studied by SDS-PAGE and fluorography. The molecular weights of bands in fluorographs was calculated relative to the migration of ${ }^{14} \mathrm{C}$-labeled protein standards.

Affinity purification studies. To show that IgG anti-BM autoantibodies in patient serum were specifically reactive with polypeptides in human keratinocyte culture media, aliquots of patient (two subjects) or control sera were affinity purified on lamina densa/dermal components of $1 \mathrm{M} \mathrm{NaCl}$ split human skin (14). In brief, strips of lamina densa/dermis were separately incubated with patient or control sera ( $1: 5$ in PBS) for $90 \mathrm{~min}$ at room temperature and then washed extensively with PBS. Anti-BM antibodies were eluted with $20 \mathrm{mM}$ sodium citrate, $\mathrm{pH} 3.2$, neutralized immediately with $2 \mathrm{M}$ Tris, $\mathrm{pH} 9.0$, dialyzed extensively against TBS for $16 \mathrm{~h}$ at $4^{\circ} \mathrm{C}$, and concentrated by ultrafiltration (Centricon 30; Amicon Corp., Danvers, MA). Affinitypurified anti-BM antibodies were analyzed in indirect immunofluorescence microscopy studies of $1 \mathrm{M} \mathrm{NaCl}$ split skin, as well as immunoprecipitation studies of radiolabeled human keratinocyte culture media.

\section{Results and Discussion}

All of our patients with blistering, erosive, and scarring lesions of mucous membranes have circulating IgG anti-BM autoantibodies that exclusively bind the dermal (rather than the epidermal) side of $1 \mathrm{M} \mathrm{NaCl}$ split skin (titers: 1:160, 1:5, and 1:40) (Fig. $1 A$ ). By immunoelectron microscopy, these patients show in situ deposits of immunoreactants and circulating IgG autoantibodies that bind the lamina lucida/lamina densa interface of epidermal BM (Fig. $1 C$ ). Moreover, autoantibodies in these patients' sera immunoprecipitate a distinct set of polypeptides from culture media of biosynthetically radiolabeled human keratinocytes. Analysis of reduced immunoprecipitation samples shows that patient sera identify polypeptides of $170,145,125$, and $95 \mathrm{kD}$ (Fig. $2 A$, lanes $1-3$ ). Under nonreducing conditions, these polypeptides remain disulfide linked, migrating as a single, large protein species $(\geq 600 \mathrm{kD}$; data not shown). To determine whether antiepidermal BM autoantibodies in patient sera (rather than some other circulating Ig species) are responsible for immunoprecipitating these polypeptides, serum samples from two patients were affinity purified on lamina densa/dermal components of epidermal BM in $1 \mathrm{M} \mathrm{NaCl}$ split skin, and then characterized in immunofluorescence and immunoprecipitation studies. Affinity-purified antiBM autoantibodies from these patients exclusively bind the dermal side of $1 \mathrm{M} \mathrm{NaCl}$ split skin and immunoprecipitate the same distinct set of polypeptides in keratinocyte culture media described above (Fig. $2 B$, lanes 2 and 3 ).

The keratinocyte polypeptides identified by these patients' circulating autoantibodies have size characteristics, disulfide linkages, and a distribution within cutaneous BMs such as those of two previously described epidermal BM lamina lucida constituents: epiligrin, the major keratinocyte integrin ligand (2); and BM600, a large keratinocyte-derived glycoprotein an- 

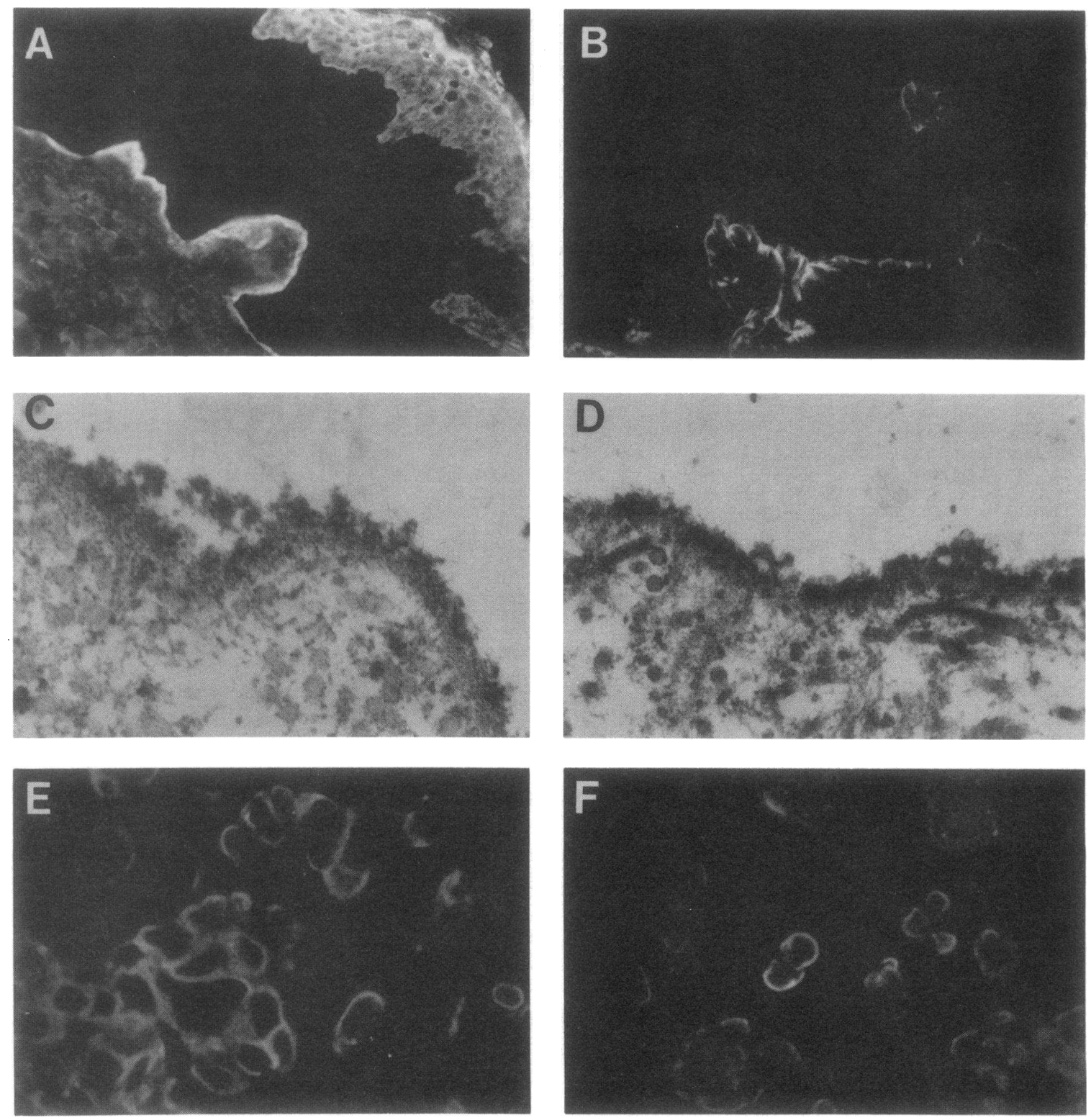

Figure 1. IgG autoantibodies in patient serum and P1El monoclonal antiepiligrin antibody bind only the dermal side of $1 \mathrm{M} \mathrm{NaCl}$ split skin in indirect immunofluorescence microscopy studies ( $A$ and $B$, respectively), and specifically localize to the lamina lucida/lamina densa interface of this test substrate by indirect immunoelectron microscopy ( $C$ and $D$, respectively). Normal human serum and control monoclonal antibody show no evidence of reactivity to $1 \mathrm{M} \mathrm{NaCl}$ split skin in these studies (data not shown). Patient serum $(E)$ and $\mathrm{P} 1 \mathrm{E} 1(F)$ (but not controls) show the same pattern of reactivity to the ECM and periphery of cultured A-431 cells by indirect immunofluorescence microscopy. Cultured human keratinocytes bind IgG autoantibodies in patient serum and P1E1 in the same manner as A-431 cells (data not shown).

tigen identified by murine monoclonal antibody GB3 (5). Interestingly, although the biological role of BM600 has never been defined, previous studies have shown that this antigen is absent in fetal skin samples from patients with LJEB (6), an inherited blistering disease characterized by a defect within the lamina lucida that results in separation of epidermis from BM. Because epiligrin and BM600 are thought to promote adherence of keratinocytes to ECM in vitro and BM in vivo, it is logical to consider their altered expression in diseases characterized by separation of epidermis from BM. To assess the identity or relatedness of epiligrin, BM600, and antigens recognized by our patients' circulating autoantibodies, comparative immunoprecipitation experiments were performed (Fig. $2 C$ ). These studies show that patient sera (Fig. $2 C$, lane 2), P1E1
(Fig. $2 C$, lane 3), and GB3 (Fig. $2 C$, lane 4 ) all recognize the same set of polypeptides in keratinocyte culture media. Moreover, preabsorption of keratinocyte culture media with patient serum (but not control normal human serum) removes all polypeptides reactive with patient autoantibodies, P1E1, or GB3 (Fig. 3, lanes 1-3; control studies, Fig. 3, lanes 4-6). These immunoprecipitation studies show that epiligrin is physically associated with the antigenic target of patient autoantibodies. Whether the autoimmune epitopes reside on epiligrin itself is yet to be determined. Patient sera and P1E1 also demonstrate (a) continuous staining of epidermal BM in normal human skin by indirect immunofluorescence microscopy (Fig. 1, $A$ and $B) ;(b)$ staining of the lamina lucida/lamina densa interface of epidermal BM by indirect immunoelectron microscopy 
(Fig. 1, $C$ and $D) ;(c)$ staining of the ECM and cell periphery of cultured human keratinocytes and A-431 cells, a human epidermoid carcinoma cell line (Fig. 1, $E$ and $F$ ); and $(d)$ complete absence of reactivity to a prenatal skin sample from a patient with LJEB (Fig. 4, $A$ and $B$ ). That epiligrin is targeted by autoantibodies in an acquired subepidermal blistering disease and absent from the skin of patients with a lethal inherited subepidermal blistering disease unify an important number of experimental and clinical observations and provide pathologic correlates that substantiate the key physiologic role(s) of epiligrin in basal keratinocyte adhesion to epidermal BM.

Our findings may also have direct relevance to kalinin, a keratinocyte-derived glycoprotein recently described by Rousselle et al. (15). Like epiligrin, kalinin consists of a set of disulfide linked polypeptides that are present in keratinocyte ECM in vitro and epidermal BM lamina lucida in vivo. Ultrastructur-
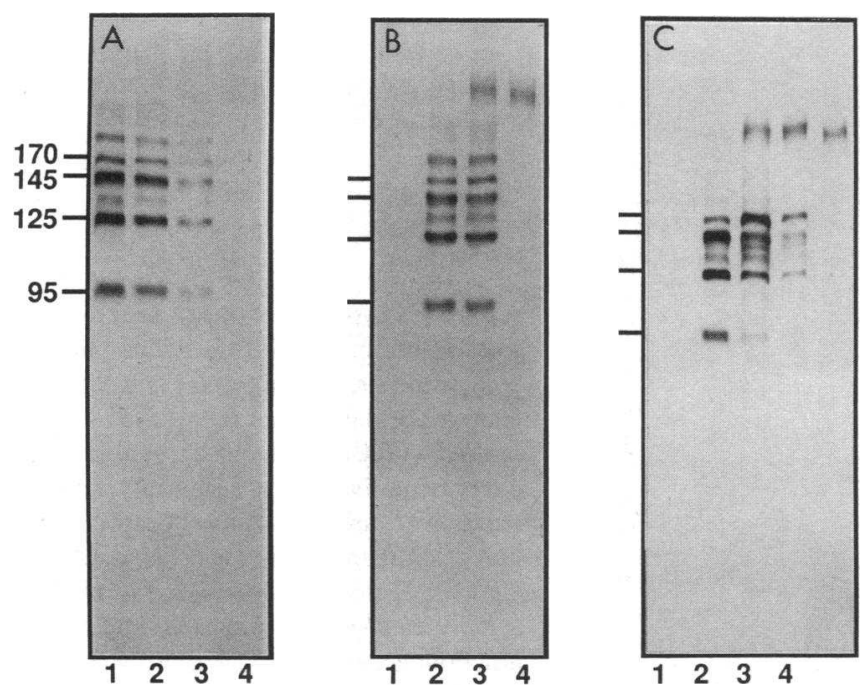

Figure 2. Patient IgG anti-BM autoantibodies immunoprecipitate a distinct set of specific polypeptides in human keratinocyte culture media that comparative studies show are identical to epiligrin and BM600. $(A)$ Analysis of reduced immunoprecipitation samples shows that patient sera (lanes 1-3) but not control normal human serum (lane 4) identify a set of disulfide linked polypeptides of 170,145 , 125 , and $95 \mathrm{kD}$ in human keratinocyte culture media; nonreduced, this set of polypeptides remain disulfide linked and migrate as a single large protein species ( $\geq 600 \mathrm{kD}$; data not shown). (B) Affinity-purified anti-epidermal BM autoantibodies in patient serum (representative sample, lane 3) identify the same distinct set of polypeptides (reducing conditions) seen in studies of patient serum (lane 2); studies of control human serum (lane 1 ) and processed control human serum (i.e., serum subjected to the same affinity purification procedures applied to patient serum, lane 4 ) show no evidence of reactivity to these polypeptides. $(C)$ Comparative immunoprecipitation studies demonstrate that patient serum (representative sample, lane 2), antiepiligrin monoclonal antibody P1E1 (lane 3), and anti-BM600 monoclonal antibody GB3 (lane 4) all recognize disulfide linked polypeptides of $170,145,125$, and $95 \mathrm{kD}$ in radiolabeled human keratinocyte culture media. Control normal human serum (lane 1) and control monoclonal antibody (lane 5) show no evidence of reactivity to these polypeptides.

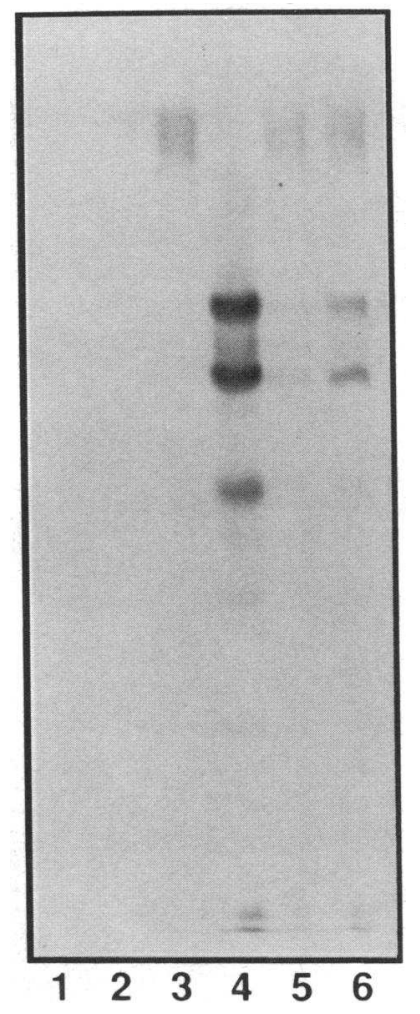

Figure 3. Biosynthetically radiolabeled human keratinocyte culture media was preabsorbed with patient serum or normal human serum (control) for $2 \mathrm{~h}$ at $4^{\circ} \mathrm{C}$, twice mixed with freshly washed Pansorbin, and then studied in immunoprecipitation experiments. Analysis of immunoprecipitation samples (reducing conditions) shows that preabsorption of keratinocyte media with patient serum removes all polypeptides reactive with patient autoantibodies (lane 1), P1E1 (lane 2), or GB3 (lane 3). In contrast, immunoprecipitation studies of keratinocyte culture media preabsorbed with normal human serum show that patient autoantibodies (lane 4), P1E1 (lane 5), and GB3 (lane 6) all identify the same disulfide linked polypeptides. (Note: Longer exposure of this fluorograph reveals the same predominant polypeptides identified in immunoprecipitation studies shown in Fig. 2.)

ally, both of these proteins are thought to reside in anchoring filaments, elements that link basal keratinocytes to lamina densa and dermal anchoring fibrils. The molecular weights of these polypeptides, their disulfide association, and their ultrastructural localization in skin suggest that kalinin and epiligrin may be the same or related glycoproteins. Moreover, studies in our laboratories have found that autoantibodies from our patients and monoclonal antiepiligrin antibody P1E1 bind human epidermal BM, but they do not react with vascular or renal BMs. These findings demonstrate that epiligrin has the same tissue distribution as previously documented for kalinin and further support the notion that these glycoproteins are identical or closely related. Interestingly, Rousselle et al. reported that skin fragments exposed to antikalinin antibodies extensively de-epithelize as a consequence of cleavage within the lamina lucida (15). While the mechanism of this detachment is unknown, it suggests that antibodies directed against key adhesive proteins in skin may directly cause separation of epidermis from BM, a finding of potential relevance to the pathogenesis of blisters seen in our patients with antiepiligrin autoantibodies.

Antiepiligrin autoantibodies serve as a specific marker for patients with this blistering skin disorder. We have not identified antiepiligrin autoantibodies in immunoprecipitation studies using serum from other patients with the cicatricial pemphigoid phenotype ( $n=5)$, numerous other patients with subepidermal blistering skin diseases (bullous pemphigoid, $n=16$; epidermolysis bullosa acquisita, $n=1$; linear IgA dermatosis, $n$ $=1$; other subepidermal bullous disease patients, $n=10)(9$, 12 ), one skin disease control patient, or five normal volunteers. These findings suggest the existence of a distinct noso- 


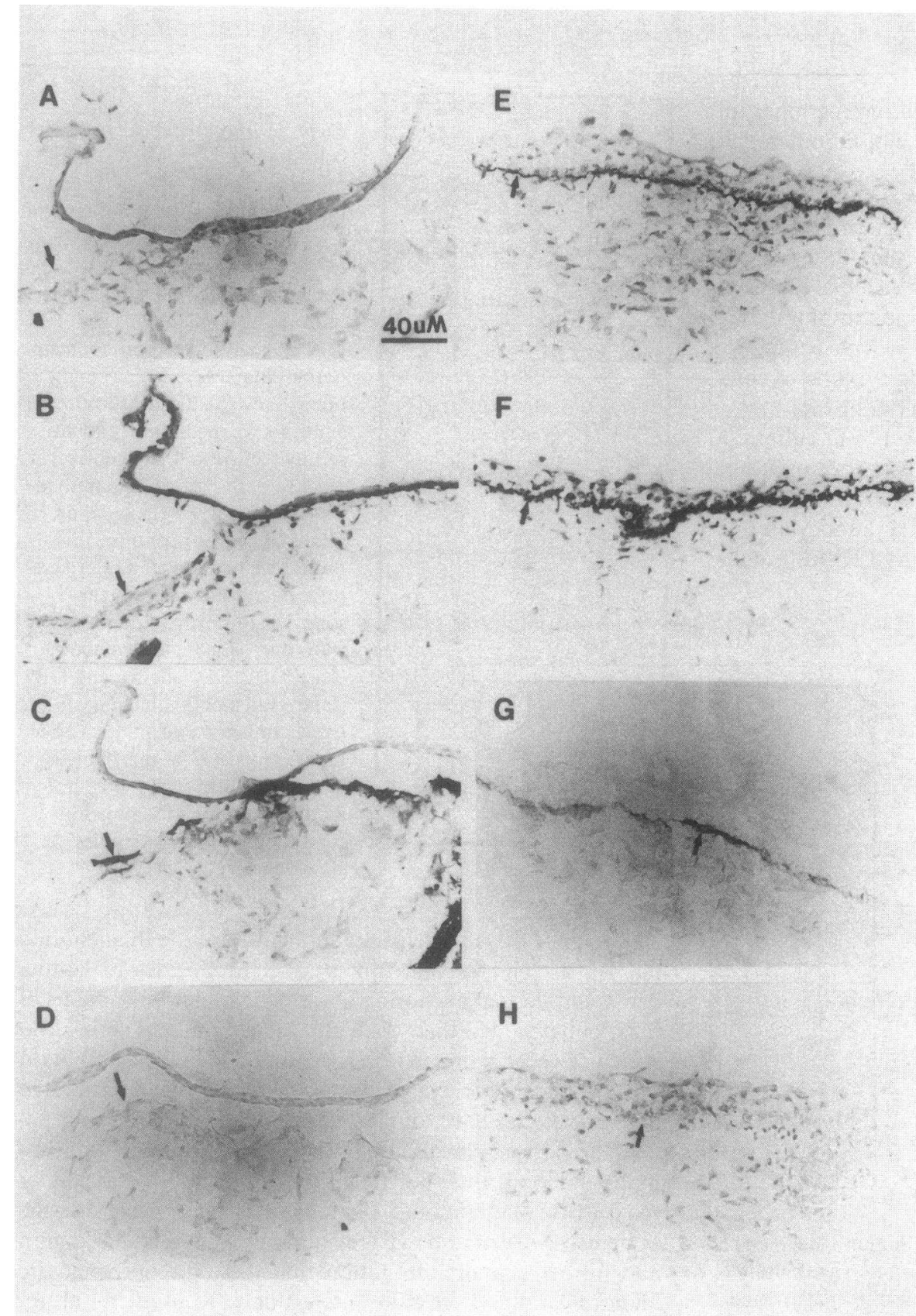

Figure 4. Cryostat sections $(6 \mu \mathrm{m})$ of skin from a human fetus with lethal junctional epidermolysis bullosa $(A-D)$ and skin from a normal human fetus $(E-H)$ were reacted with $\mathrm{PlEl}(A$ and $E)$, serum from a representative patient $(B$ and $F$ ), antitenascin ( $C$ and $G$; positive control), and normal human serum ( $D$ and $H$; negative control). Tenascin is expressed in the epidermal BM of both normal and lethal junctional epidermolysis bullosa skin ( $G$ and $C$, respectively; see arrows that indicate epidermal BM). In contrast, P1E1 and patient serum stain the epidermal $\mathrm{BM}$ in normal ( $E$ and $F$, respectively) but not diseased ( $A$ and $B$, respectively) skin.

logic entity characterized by mucosal predominant subepidermal blistering lesions and antiepiligrin autoantibodies. The lack of expression of epiligrin in patients with LJEB serves as another important example of how an abnormality of epiligrin is associated with separation of epidermis from BM. As the key integrin ligand for $\alpha_{3} \beta_{1}$ in plasma membranes and focal adhesions, epiligrin has been shown to play a key role in keratinocyte adhesion in vitro. Findings in this report identify two disease states that attest to the importance of epiligrin in maintaining adhesion of epidermis to BM in human skin in vivo. Knowledge about the gene (or genes) that encodes this molecule (or molecules), as well as factors that govern its (or their) expression are of great importance in gaining a better understanding of epidermal structure and morphogenesis.

\section{Acknowledgments}

The authors thank Dr. Thomas Shepard and Dr. Alan Fantel for providing normal human fetal tissue through the Laboratory for the Study of Human Embryos and Fetuses (National Institutes of Health Grant HD-00836; University of Washington, Seattle, WA); Dr. Virginia Sybert, Dr. Karen Holbrook, and Dr. Lynn Smith (University of Washington), as well as.Dr. Rena Folk (Children's Hospital of Los Angeles, Los Angeles, CA) for providing tissue from junctional epidermolysis bullosa patients; Dr. John R. Stanley, Dr. Stephen I. Katz, and Dr. Douglas R. Lowy for their review of this material, and Ms. Fran Ford for typing this manuscript.

This work was supported in part by National Institutes of Health (NIH) grant AR-37446 to K. B. Yancey, the Sulzberger Fund Endowment to K. B. Yancey and N. Domloge-Hultsch, as well as American 
Cancer Society grant CD-453F and NIH grants RO1-CA49259 and 5 PO1 AR-21557 to W. G. Carter.

The opinions or assertions contained in this paper are the private ones of the authors and are not to be construed as official or reflecting the views of the Department of Defense or the Uniformed Services University of the Health Sciences.

\section{References}

1. Carter, W. G., E. A. Wayner, T. S. Bouchard, and P. Kaur. 1990. The role of integrins $\alpha_{3} \beta_{1}$ in cell-cell and cell-substrate adhesion of human epidermal cells. $J$. Cell Biol. 110:1387-1404.

2. Carter, W. G., M. C. Ryan, and P. J. Gahr. 1991. Epiligrin, a new cell adhesion ligand for integrin $\alpha_{3} \beta_{1}$ in epithelial basement membranes. Cell. 65:599-610.

3. Verrando, P., B.-L. Hsi, C.-J. Yeh, A. Pisani, N. Serieys, and J.-P. Ortonne. 1987. Monoclonal antibody GB3, a new probe for the study of human basement membranes and hemidesmosomes. Exp. Cell Res. 170:116-128.

4. Verrando, P., C. Blanchet-Bardon, A. Pisani, L. Thomas, F. Cambazard, R. A. Eady, O. Schofield, and J.-P. Ortonne. 1991. Monoclonal antibody GB3 defines a widespread defect of several basement membranes and a keratinocyte dysfunction in patients with lethal junctional epidermolysis bullosa. Lab. Invest. 64:85-92.

5. Katz, S. I. 1984. The epidermal basement membrane zone-structure, ontogeny, and role in disease. J. Am. Acad. Dermatol. 11:1025-1037.

6. Fine, J. D., G. R. Neises, and S. I. Katz. 1984. Immunofluorescence and immunoelectron microscopic studies in cicatricial pemphigoid. J. Invest. Dermatol. 82:39-43.
7. Basset-Seguin, N., S. W. Caughman, and K. B. Yancey. 1990. A-431 cells and human keratinocytes synthesize and secrete the third component of complement. J. Invest. Dermatol. 95:621-625.

8. Gammon, W. R., R. A. Briggaman, A. O. Inman, L. L. Queen, and C. E. Wheeler. 1984. Differentiating anti-lamina lucida and anti-sublamina densa antiBMZ antibodies by indirect immunofluorescence on $1.0 \mathrm{M}$ sodium chloride-separated skin. J. Invest. Dermatol. 82:139-144.

9. Domloge-Hultsch, N., P. Bisalbutra, W. R. Gammon, and K. B. Yancey. 1991. Direct immunofluorescence microscopy of $1 \mathrm{~mol} / \mathrm{L}$ sodium chloridetreated patient skin. J. Am. Acad. Dermatol. 24:946-951.

10. Sternberger, L. A., P. A. Hardy, J. J. Cuculis, and H. G. Meyer. 1970. The unlabeled antibody enzyme method of immunohistochemistry: preparation and properties of soluble antigen: antibody complex (horseradish peroxidase-antihorseradish peroxidase), and its use in identification of spirochetes. J. Histochem. Cytochem. 18:315-333.

11. Holubar, K., K. Wolff, K. Konrad, and E. G. Beutner. 1975. Ultrastructural localization of immunoglobulins in bullous pemphigoid skin. Employment of a new peroxidase-antiperoxidase method. J. Invest. Dermatol. 64:220-225.

12. Domloge-Hultsch, N., L. Utecht, W. James, and K. B. Yancey. 1990. Autoantibodies from patients with localized and generalized bullous pemphigoid immunoprecipitate the same $230-\mathrm{kD}$ keratinocyte antigen. Arch. Dermatol. 126:1337-1341.

13. Stanley, J. R., P. Hawley-Nelson, S. H. Yuspa, E. F. Shevach, and S. I. Katz. 1981. Characterization of bullous pemphigoid antigen: a unique basement membrane protein of stratified squamous epithelia. Cell. 24:897-903.

14. Zone, J. J., T. B. Taylor, D. P. Kadunce, and L. J. Meyer. 1990. Identification of the cutaneous basement membrane zone antigen and isolation of antibody in linear immunoglobulin A bullous dermatosis. J. Clin. Invest. 85:812-820.

15. Rousselle, P., G. P. Lunstrum, D. R. Keene, and R. E. Burgeson. 1991. Kalinin: an epithelium-specific basement membrane adhesion molecule that is a component of anchoring filaments. J. Cell Biol. 114:567-576. 